\title{
Currents' Physical Components (CPC) concept in wind farm harmonic current studies
}

\author{
L. Sainz ${ }^{1}$ and J. Cunill-Solà ${ }^{2}$ \\ ${ }^{1}$ Department of Electrical Engineering \\ E.T.S.E.I.B, Technical University of Catalonia (UPC) \\ Av. Diagonal 647, 08028 Barcelona (Spain) \\ Phone/Fax number:+0034 93 4011759/+0034 93 4017433, e-mail: sainz@ee.upc.edu \\ ${ }^{2}$ Department of Electrical Engineering \\ E.P.S.E.M, Technical University of Catalonia (UPC) \\ Av. de les Bases 61-73, 08240 Manresa (Spain) \\ Phone/Fax number:+0034 93 8777263/+0034 93 8777202, e-mail: cunill@ee.upc.edu
}

\begin{abstract}
Very little information about actual wind farm harmonic measurements is available in the literature. This paper analyzes the harmonic measurements of an $18 \mathrm{MW}$ wind farm with the CPC concept to determine if they are consumed or injected by the wind farm and to study the different reactive power definitions. The CPC concept is an approach to power theory that provides a physical interpretation of power phenomena in electrical systems with linear, time-invariant (LTI) loads and harmonic generating loads (HGLs) such as wind farms.
\end{abstract}

\section{Key words}

Wind power generation, harmonics, power theory.

\section{Introduction}

The increasing number of wind farms worldwide causes power quality problems such as harmonic current emissions [1]-[6]. These emissions produce voltage distortion in networks, and their measurement and inclusion in WT power certificates are therefore required by current standards [7], [8]. Knowledge of these emissions is fundamental to study the influence of wind farms on network harmonic distortion. Although they are a well-known topic, very few studies based on actual measurements have been published [2]-[5]. Recently, the harmonic current behavior of an $18 \mathrm{MW}$ wind farm was investigated from a large number of measurements in [9]. The wind farm harmonic emissions were studied, and the influence of the wind farm working point on these emissions was extensively analyzed. In addition, the probability density functions of the harmonic magnitudes and phase angles were discussed and compared in detail with analytical and empirical distributions in the literature.

Wind farm harmonics and, in particular, power transmission phenomena can be analyzed by the CPC concept. The CPC concept is currently the most advanced form of power theory of electric systems with periodic and semi-periodic voltages and currents. It explains power properties of single- and three-phase systems with LTI loads and HGLs [10]-13].

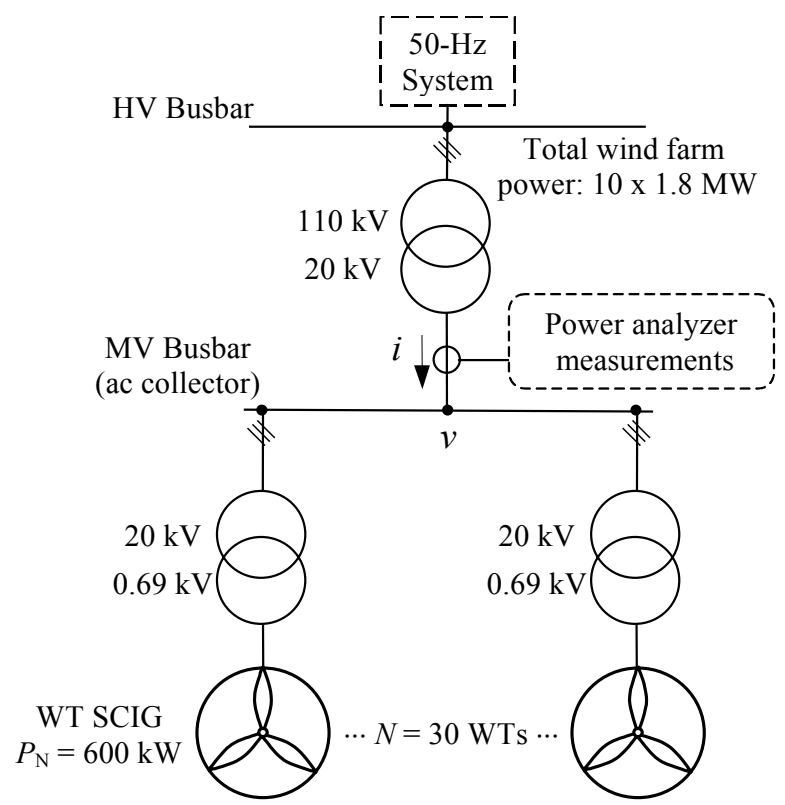

Fig. 1. One-line diagram of the wind farm.

The paper analyzes the harmonic current measurements in [9] and related power phenomena by the CPC concept.

\section{Wind Farm Measurements}

\section{A. Wind Farm}

The harmonic currents of the wind farm in the one-line diagram of Fig. 1 were extensively studied in [9]. The farm consists of 30x600 kW WT squirrel-cage induction generators (SCIGs) connected to the MV collector with $20 / 0.69 \mathrm{kV}$ transformers. The rated power of the wind farm is $P_{\mathrm{N}}=18 \mathrm{MW}$ and the rated current at the MV collector is $I_{\mathrm{N}}=522 \mathrm{~A}$. A $110 / 20 \mathrm{kV}$ transformer substation connects the MV and HV busbars.

\section{B. Harmonic current measurements}

Measurements were carried out at the wind farm MV collector (Fig. 1) with the power network analyzer AR5-L of CIRCUTOR (Fig. 2) according to the requirements of IEC 61400-21 [7] and IEC 61000-4-7 [8] standards. The analyzer has a $0.5 \%$ voltage and current 


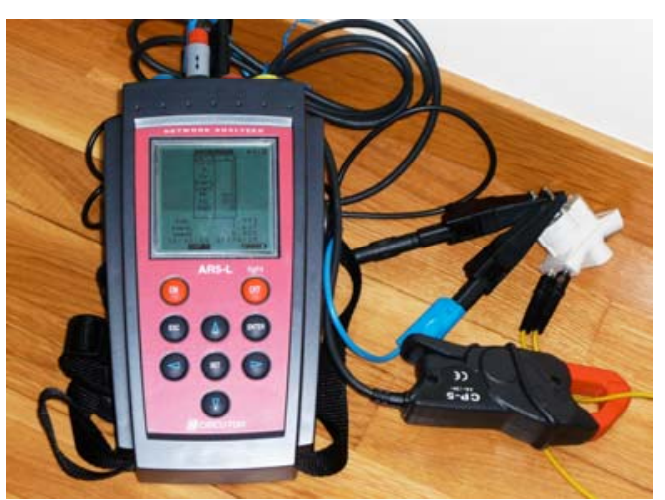

Fig. 2. Power network analyzer AR5-L of CIRCUTOR.

accuracy class, a sampling frequency sufficient to evaluate up to the $63^{\text {rd }}$ harmonic order and a $1 \mathrm{Mb}$ onboard memory to save all measured or calculated parameters for future retrieving. The current clamps employed in the measurements have a total full-scale accuracy of $1 \%$ with a bandwidth of $10 \mathrm{~Hz}$ to $5 \mathrm{kHz}$. The active power, $P$, and voltage and current waveforms, $v$ and $i$, respectively, were recorded over a 6-day period with a 10-minute time interval between readings, each record being the 10 -min average value. The long storage period made it possible to record data for the whole power range of the wind farm, and the 10-min average values provided sufficient accuracy of the voltage and current measurements. All the recorded voltage and current values were stored on a hard disk and treated with customized MATLAB software for harmonic analysis. Thus, assuming that the magnitude of the currents is stationary, the Fourier transform was applied to a $20-\mathrm{ms}$ rectangular window providing a $50-\mathrm{Hz}$ frequency resolution.

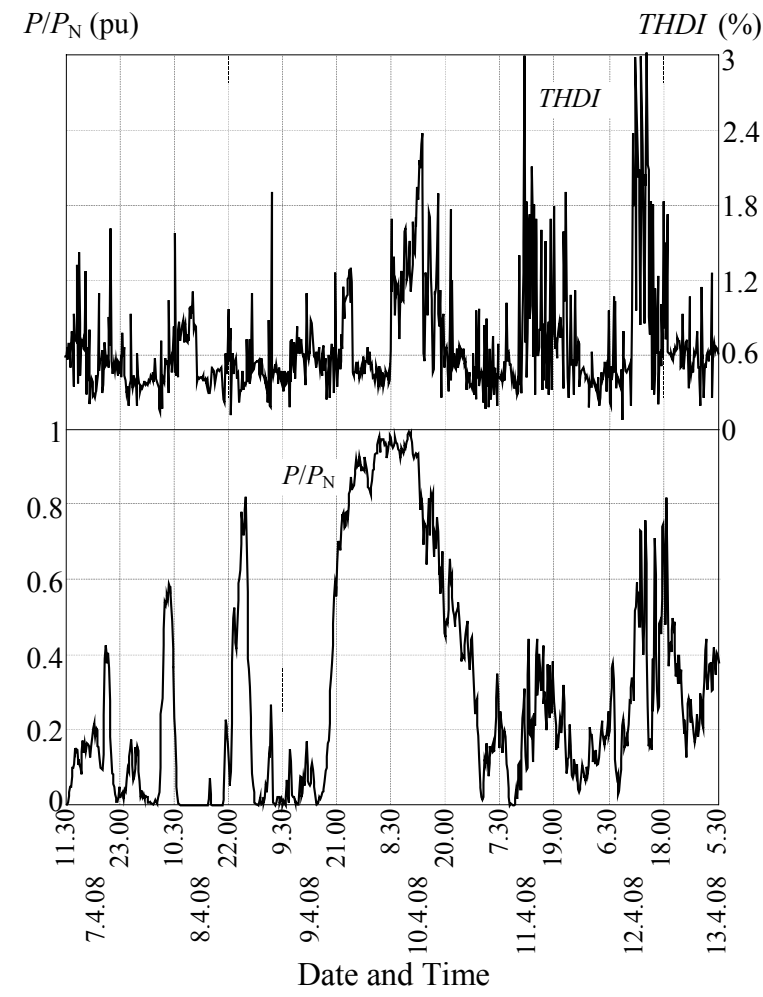

Fig. 3. Time course of the total harmonic distortion and output power ratio.
THDI (\%)

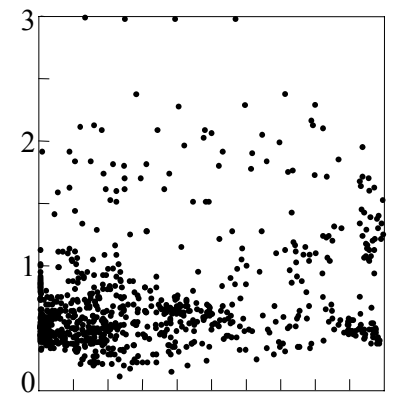

$\mathrm{HDI}_{5}(\%)$

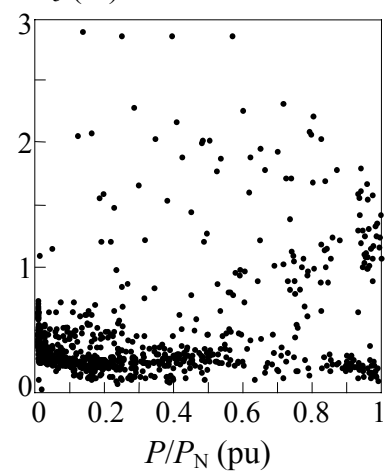

$H D I_{1}(\%)$

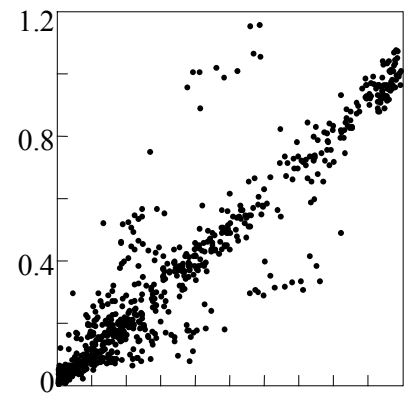

$\mathrm{HDI}_{7}(\%)$

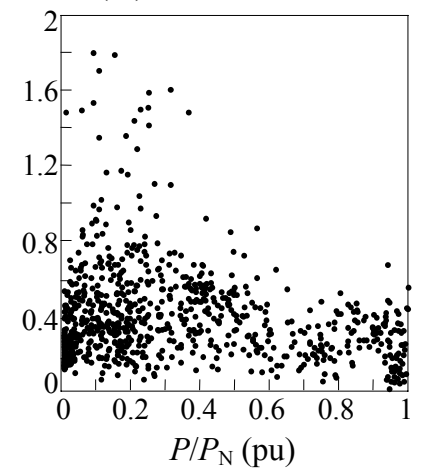

Fig. 4. Wind farm harmonic distortion versus output power for the total measurement period course.

The current and voltage harmonics $\left(\underline{v}_{h}\right.$ and $\underline{i}_{h}$, respectively) were evaluated up to the fiftieth order (2.5 kHz for $50-\mathrm{Hz}$ systems):

$$
x(t) \Rightarrow \underline{x}_{h}=x_{h} \angle \phi_{x_{h}} \quad h=1, \ldots, 50 \quad(x=v, i) .
$$

The wind farm harmonic currents and the influence of the wind farm working point on them were analyzed. In addition, scatter plots of the harmonics (magnitude and phase angle) versus the wind farm output power were provided and the random behavior of the harmonics was examined from the experimental measurements. It could be observed that the dominant harmonics belonged to the low-order set (in particular, the highest were $5^{\text {th }}$ and $7^{\text {th }}$ ) and that a high-order harmonic pattern existed in the current spectrum between 1.0 and $2.0 \mathrm{kHz}$. A summary of the measurements in [9] is given in Fig. 3 to Fig. 5 considering the total and individual harmonic current distortions as follows [7]:

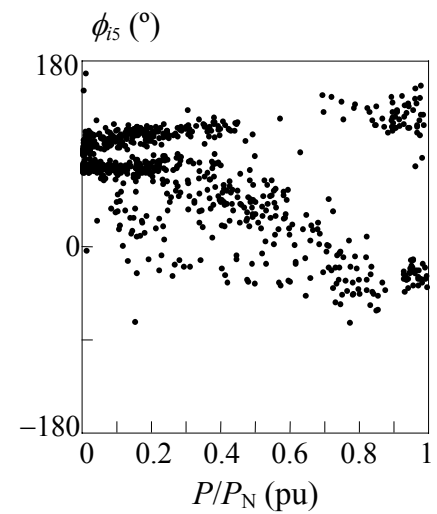

$$
\phi_{i 7}\left({ }^{\circ}\right)
$$

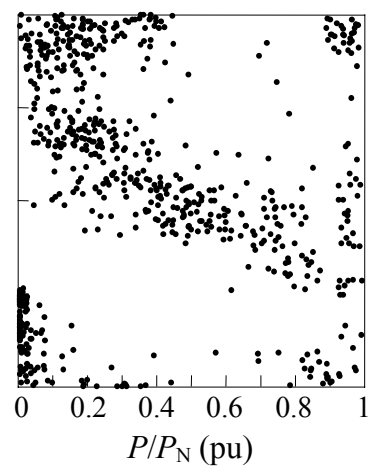

Fig. 5. Phase angles of the wind farm $5^{\text {th }}$ and $7^{\text {th }}$ harmonic currents versus output power for the total measurement period course. 


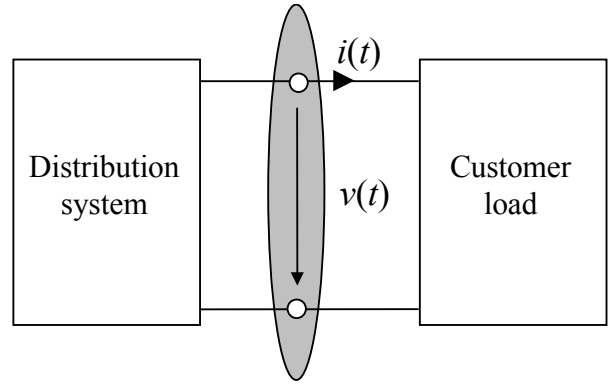

Fig. 6. Cross-section between distribution system and customer load.

$$
T H D I=\frac{\sqrt{\sum_{h=2}^{50} i_{h}^{2}}}{I_{\mathrm{N}}}, \quad H D I_{h}=\frac{i_{h}}{I_{\mathrm{N}}} \quad(h=1,3 \ldots),
$$

where $I_{\mathrm{N}}$ is the wind farm rated current and $i_{h}$ the fundamental and harmonic magnitudes of the current at the MV collector. Fig. 3 shows the time course of the $T H D I$ and the wind farm output power. Fig. 4 shows the scatter plot of the THDI and $H D I_{h}(h=1,5$ and 7$)$ versus the wind farm output power. Fig. 5 contains the phase angle scatter plots of the most significant harmonic currents (i.e., $5^{\text {th }}$ and $7^{\text {th }}$ harmonics) versus the output power. The following remarks on the measurements can be made:

- The fundamental current increases proportionally with the wind farm output power while the harmonic currents vary stochastically.

- In spite of the random behavior of wind farm harmonic currents, these are usually modeled as fixed current injections due to their small variation with respect to the wind farm operating point. In accordance with IEC 61000 standards, the magnitudes adopted for harmonic current injections are generally the $95 \%$ non-exceeding probability values of these currents.

- Deterministic models are usually based on the arithmetic sum of the average or the $95 \%$ percentile values of measured wind farm harmonic currents. These models are the simplest ones and compensate for the lack of information about harmonic current phase angles. Nevertheless, they lead to overestimation of system harmonic distortion because the random variation of harmonics (in particular their phase angles) is disregarded.

In [9], a complete study of the wind farm harmonic current stochastic behavior was performed from the field measurements.

The analysis of the wind farm harmonic measurements in [9] can be theoretically supported with the CPC concept. In particular, the origin of the harmonic currents measured in the above study can be analyzed (i.e. wether these currents are consumed or injected by the wind farm). The CPC concept is an advanced form of power theory systems with non-sinusoidal voltage and currents [10]-[13]. Thus, the next Section summarizes some of the power theory concepts introduced by the CPC approach in the presence of HGL [12].

\section{CPC in Single-Phase Circuits with HGLs}

Let us consider the single-phase network in Fig. 6, formed by two subnetworks, the distribution system and the customer load, and the voltage and current, $v(t)$ and $i(t)$, measured at the cross-section between the distribution system and the customer load. If $v(t)$ and $i(t)$ belong to the linear space $L_{T}^{2}$, i.e.

$$
x(t)=x(t \pm k T), \quad \frac{1}{T} \int_{0}^{T} x^{2}(t) d t<\infty \quad(x=v, i),
$$

where $k$ is any integer number and $T$, called the period of $x(t)$, is a nonzero real number, they can be expressed with the Fourier series:

$$
x(t)=X_{0}+\sqrt{2} \operatorname{Re} \sum_{h=1}^{\infty} \underline{X}_{h} e^{j \omega_{h} t}=\sum_{h \in \mathrm{H}} x_{h}(t) \quad(x=v, i),
$$

where $x_{h}(t)$ is the harmonic of order $h$ of the quantity $x(t)$, $\mathrm{H}$ represents the set of harmonic orders $h$, including $h=0$, of harmonics with nonzero complex rms values

$$
\underline{X}_{h}=X_{h} e^{j \alpha_{X}}
$$

The instantaneous power at the cross-section in Fig. 6 is the rate of electric energy $W(t)$ flow from the distribution system to the customer load and can be expressed as a sum of harmonics as follows:

$$
p(t)=\frac{d W(t)}{d t}=v(t) i(t)=\sum_{h \in \mathrm{H}} v_{h}(t) \sum_{h \in \mathrm{H}} i_{h}(t) .
$$

The active power is the average value of the instantaneous power over a single period of the voltage and current:

$$
\begin{aligned}
& P=\overline{p(t)}=\frac{1}{T} \int_{0}^{T} v(t) i(t)=\operatorname{Re} \sum_{h \in \mathrm{H}} \underline{V}_{h} \underline{I}_{h}^{*}= \\
& =\sum_{h \in \mathrm{H}} V_{h} I_{h} \cos \varphi_{h}=\sum_{h \in \mathrm{H}} P_{h} \quad\left(\varphi_{h}=\alpha_{V_{h}}-\alpha_{I_{h}}\right),
\end{aligned}
$$

where

$$
P_{h}=V_{h} I_{h} \cos \varphi_{h}
$$

is the active power of the $h^{\text {th }}$ harmonic order.

The apparent power is defined as the product of the voltage and current rms values:

$$
\|x(t)\|=\sqrt{\sum_{h \in \mathrm{H}} X_{h}^{2}} \quad(x=v, i)
$$




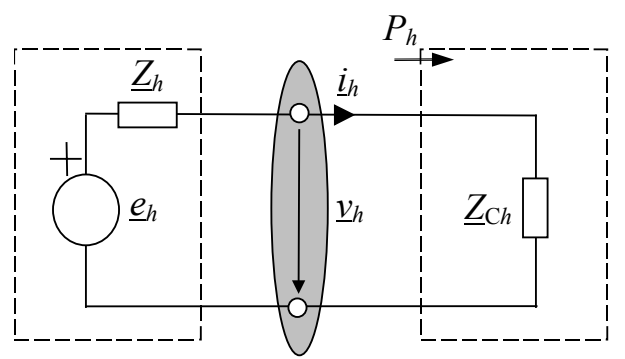

(a)

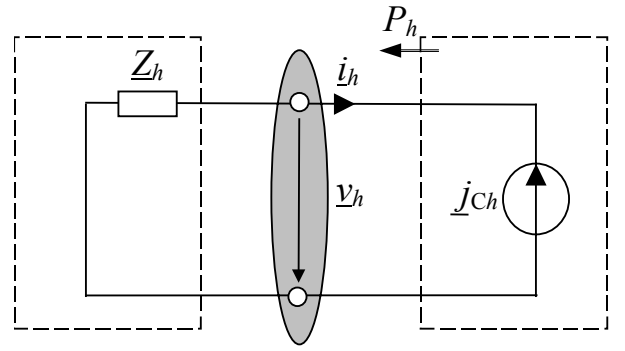

(b)

Fig. 7. Equivalent circuits: a) For harmonics $h \in \mathrm{H}_{\mathrm{A}}$. b) For harmonics $h \in \mathrm{H}_{\mathrm{B}}$.

and it can be expressed as follows:

$$
\begin{aligned}
& S=\|v(t)\|\|i(t)\|= \\
& =P^{2}+\frac{1}{2} \sum_{r \in \mathrm{H}} \sum_{s \in \mathrm{H}} V_{r}^{2} V_{s}^{2}\left(\frac{P_{s}}{V_{s}^{2}}-\frac{P_{r}}{V_{r}^{2}}\right)^{2}+\|v\|^{2} \sum_{h \in \mathrm{H}}\left(\frac{Q_{h}}{V_{h}}\right)^{2}=(10) \\
& =P^{2}+D_{s}^{2}+Q^{2},
\end{aligned}
$$

where $D_{s}$ and $Q$ are the scattered and reactive power introduced by L. S. Czarnecki [13] and

$$
Q_{h}=V_{h} I_{h} \sin \varphi_{h}
$$

is the reactive power of the $h^{\text {th }}$ harmonic order.

The power definitions introduced in (10) by the CPC concept provide better understanding of energy flow and power phenomena in electric systems and study procedures for the enhancement of power transmission efficiency and quality. The errors in the definitions introduced in power theory by different authors have also been clarified from the CPC concept, for example the definition of reactive and distortion power introduced in 1927 by Budeanu and supported by the IEEE Standard Dictionary [14]:

$$
Q_{B}=\sum_{h \in \mathrm{H}} V_{h} I_{h} \sin \varphi_{h}, \quad D_{B}=\sqrt{S^{2}-P^{2}-Q_{B}^{2}}
$$

The physical phenomena that characterize the harmonic energy flows in non-sinusoidal conditions at the crosssection in Fig. 6 can be analyzed from the phase angle $\varphi_{h}$ between the voltage and the current harmonics, $\underline{v}_{h}$ and $i_{h}$, at the cross-section, or from the harmonic active power $P_{h}(8)$. Thus, if $\left|\varphi_{h}\right| \leq \pi / 2$, there is an average component

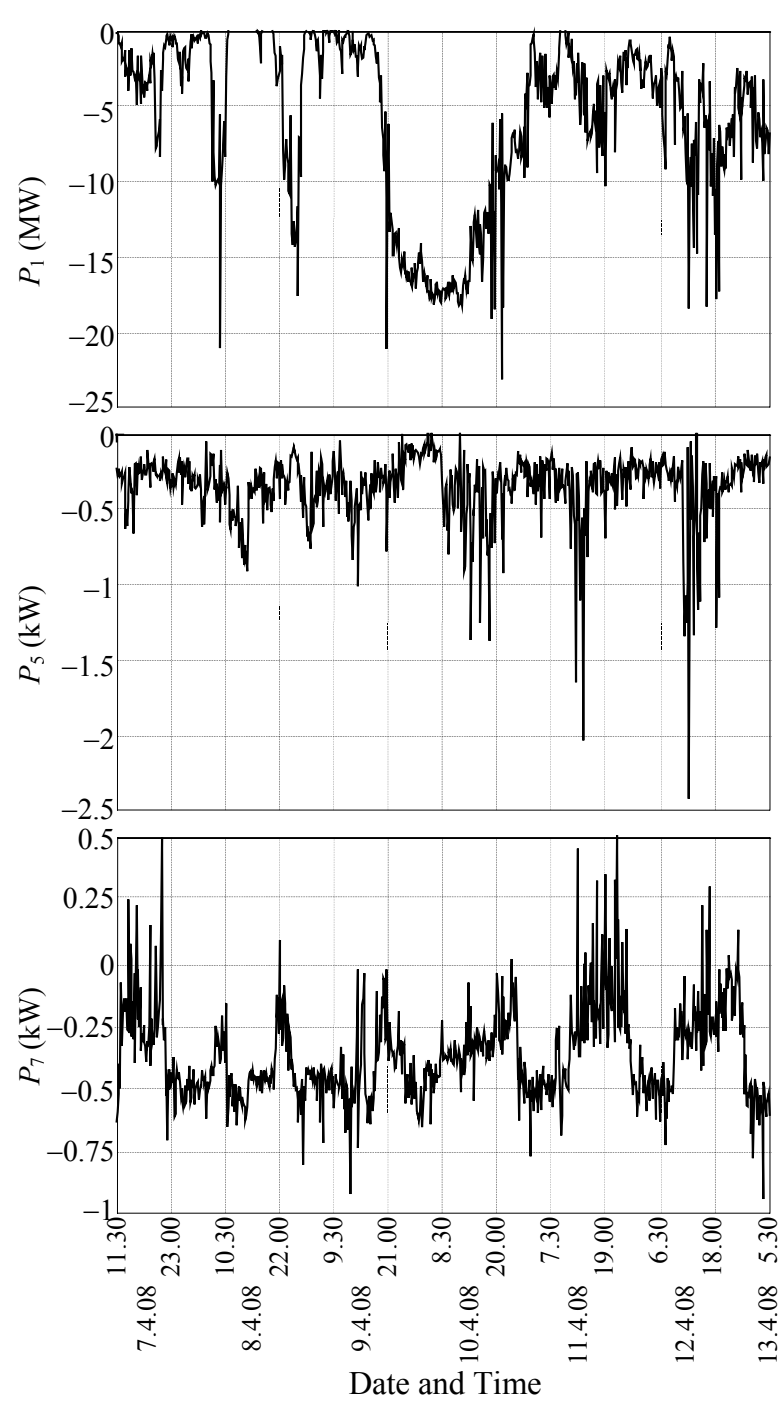

Fig. 8. Time course of the fundamental, fifth and seventh harmonic active powers.

of energy flow at the $h^{\text {th }}$ harmonic order from the distribution system towards the customer load, i.e. $P_{h} \geq 0$. If $\left|\varphi_{h}\right|>\pi / 2$, there is an average component of energy flow at the $h^{\text {th }}$ harmonic order from the customer load back the distribution system, i.e. $P_{h}<0$. Considering the previous analysis, the set $\mathrm{H}$ of all the harmonic orders can be decomposed into sub-sets $\mathrm{H}_{\mathrm{A}}$ and $\mathrm{H}_{\mathrm{B}}$ $\left(\mathrm{H}_{\mathrm{A}} \cup \mathrm{H}_{\mathrm{B}}=\mathrm{H}\right)$ as follows

$$
\begin{array}{llll}
h \in \mathrm{H}_{\mathrm{A}} & \text { if } \quad\left|\varphi_{h}\right| \leq \frac{\pi}{2} \quad \text { or } \quad P_{h} \geq 0, \\
h \in \mathrm{H}_{\mathrm{B}} \quad \text { if } & \left|\varphi_{h}\right|>\frac{\pi}{2} \quad \text { or } \quad P_{h}<0,
\end{array}
$$

and the voltage and currents can be expressed as

$$
x(t)=\sum_{h \in \mathrm{H}} x_{h}(t)=\sum_{h \in \mathrm{H}_{\mathrm{A}}} x_{h}(t)+\sum_{h \in \mathrm{H}_{\mathrm{B}}} x_{h}(t) \quad(x=v, i) .
$$

That is, the consumer load can be considered as a receiver or a harmonic source for the harmonics belonging to the sub-set $\mathrm{H}_{\mathrm{A}}$ or $\mathrm{H}_{\mathrm{B}}$, respectively. This 

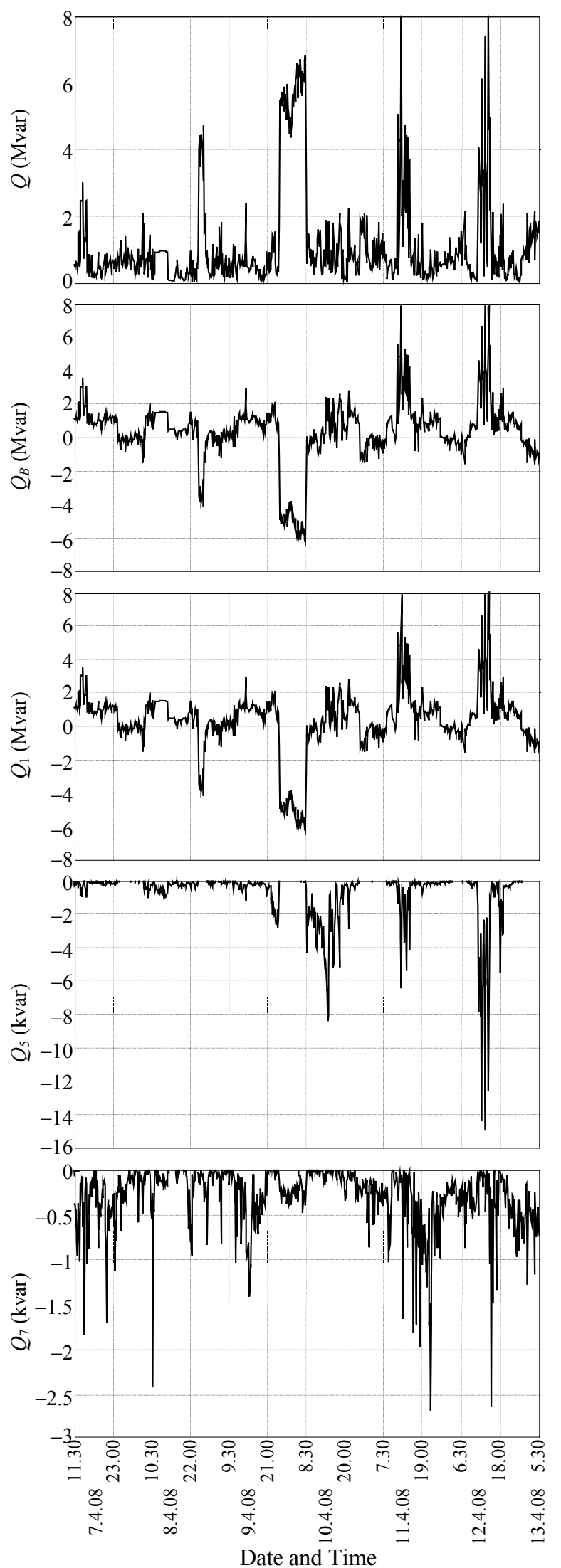

Fig. 9. Time course of the reactive powers.

decomposition allows the network in Fig. 6 to be described as a superposition of the two networks in Fig. 7 since sub-sets $\mathrm{H}_{\mathrm{A}}$ and $\mathrm{H}_{\mathrm{B}}$ do not contain common harmonic orders $h\left(\mathrm{H}_{\mathrm{A}} \cap \mathrm{H}_{\mathrm{B}}=0\right)$ and the voltage and currents belonging to them are mutually orthogonal. In Fig. 7(a) and Fig. 7(b), the customer load is modeled as an LTI load and a harmonic current source, respectively, and the distribution system is modeled as a harmonic voltage source and a passive energy receiver, respectively.

\section{CPC in Wind Farm Study}

\section{A. Active power study}

As mentioned in the previous Section, the sign of the harmonic active powers $P_{h}$ (8) allows determining whether the wind farm harmonic currents occur because of the supply harmonic voltages or they are generated in the wind farm. Thus, Fig. 8 shows the time course of the fundamental and the most significant harmonic active powers measured at the MV collector of the wind farm. From this figure, it can be noted that

- The active fundamental power $P_{1}$ flows from the wind farm back the distribution system because its sign is negative. This power nearly coincides with the output power in Fig. 3 because the harmonic power contribution is small.

- The active fifth and seventh harmonic powers $P_{5}$ and $P_{7}$ flow from the wind farm back the distribution system because their sign is negative, too. This means that the measured harmonic currents are generated by the wind farm.

\section{B. Reactive power study}

Fig. 9 shows the time course of the reactive powers calculated from the definitions presented in the previous Section [Czarnecki, harmonic and Budeanu reactive powers, i.e. (10), (11) and (12)]. From this figure, it can be noted that

- The Czarnecki reactive power $Q$ is always positive and its cancellation is achieved only when all harmonic reactive powers are cancelled.

- The Budeanu reactive power $Q_{\mathrm{B}}$ is simply the sum of the harmonic reactive powers of all harmonics and its cancellation does not necessarily mean the cancellation of such harmonic powers. In fact, the physical meaning of the harmonic reactive powers [i.e., the amplitude of the alternating component of the instantaneous power (6)] is lost in the Budeanu definition.

- This fundamental reactive power $Q_{1}$ nearly coincides with the Budeanu reactive power because the harmonic power contribution is small. This fundamental power corresponds to an inductive consumer and does not change significantly over the time course, i.e. with the different wind farm output powers.

\section{Conclusion}

The paper presents the measurements of SCIGs wind farm harmonic behavior and analyzes the power harmonic phenomena from these measurements and the CPC concept. The harmonic current injected from the wind farm back the distribution system is demonstrated with the observation of the active harmonic power sign. 
The reactive power is calculated from its different definitions and the results are discussed and compared.

\section{Acknowledgement}

This research is carried out with the financial support of grant DPI2010-15448, which the authors gratefully acknowledge.

\section{References}

[1] S. A. Papathanassiou and M. P. Papadopoulos, "Harmonic analysis in a power system with wind generation", IEEE Trans. on Power Delivery, Vol. 21, No. 4, October 2006, pp. 2006-2016.

[2] S. T. Tentzerakis and S. A. Papathanassiou, "An investigation of the harmonic emissions of wind turbines", IEEE Trans. on Energy Conversion, Vol. 22, No. 1, March 2007, pp. 150-158.

[3] D. Schulz and R. E. Hanitsch, "Investigation of the current harmonic parameters of wind energy converters", in Proc. of the IEEE Power Tech. Conference 2003, Vol. 3, June 2003.

[4] T. Thiringer, T. Petru and C. Liljegren, "Power quality impact of a sea located hybrid wind park", IEEE Trans. on Energy Conversion, Vol. 16, No. 2, June 2001, pp. 123-127.

[5] J. I. Herrera, T. W. Reddoch and J. S. Lawler, "Harmonics generated by two variable speed wind generating systems", IEEE Trans. on Energy Conversion, Vol. 3, No. 2, June 1988, pp. 267-273.

[6] J. Balcells and D. Gonzalez, "Harmonics due to resonance in a wind power plant", in Proc. of the IEEE ICHQP 1995, pp. 896-899.
[7] IEC 61400-21, Wind Turbine Generator Systems, Part 21: Measurement and Assessment of Power Quality Characteristics of Grid Connected Wind Turbines, 1st ed., December 2001.

[8] IEC 61000-4-7, Electromagnetic Compatibility (EMC) - Part 4-7: Testing and Measurement Techniques-General Guide on Harmonics and Interharmonics Measurements and Instrumentation, for Power Supply Systems and Equipment Connected Thereto, 2nd ed. August 2001.

[9] L. Sainz, J.J. Mesas, R. Teodorescu, P. Rodriguez, "Deterministic and stochastic study of wind farm harmonic currents", IEEE Trans. on Energy Conversion, accepted for publication (D.O.I.: 10.1109/TEC.2010.2045379).

[10] L. S. Czarnecki, "What is wrong with the Budeanu concept of reactive anddistortion power and why it should be abandoned", IEEE Trans. on Instrumentation and Measurement, Vol. IM-36, No. 3, September 1987, pp. 834-837.

[11] L. S. Czarnecki, "Currents' Physical Components (CPC) in circuits with nonsinusoidal voltages and currents. Part 1: Single-phase linear circuits", Electrical Power Quality and Utilization Journal, Vol. XI, No. 2, 2005, pp. 27-48.

[12] L. S. Czarnecki, "Powers in nonsinusoidal networks: Their interpretation, analysis, and measurement", IEEE Trans. on Instrumentation and Measurement, Vol. 39, No. 2, April 1990, pp. 340-345.

[13] L. S. Czarnecki, "Scattered and reactive current, voltage and power in circuits with nonsinusoidal waveforms and their compensation", IEEE Trans. on Instrumentation and Measurement, Vol. 40, No. 3, June 1991, pp. 563-567.

[14] The New IEEE Standard Dictionary of Electrical and Electrotecnics Terms, IEEE, Inc., New York 1997. 